\title{
Neuropathic bladder and its sequelae: a management challenge to the Paediatrician
}

\author{
Perera RMS ${ }^{1}$, Lokuarachchi Sandya ${ }^{2}$ \\ ${ }^{1}$ Registrar in Paediatrics, ${ }^{2}$ Consultant Paediatrician, Teaching Hospital, Karapitiya, Galle.
}

\section{Introduction}

Neuropathic bladder commonly occurs secondary to congenital anomalies of the lower spine [1]. It can lead to recurrent urinary tract infections (UTI), vesicoureteric reflux (VUR) and hydronephrosis resulting in end stage renal failure. Prompt recognition and management improve the prognosis [1].

\section{Case Report}

A 7 month-old baby girl with a history of a repaired lumbosacral meningomyelocele at the age of four weeks, presented with fever, refusal of feeds, poor cry and reduced urine output for three days. She had been given a short course of antibiotics by a general practitioner but her symptoms persisted.

She had a culture positive UTI with ultrasound evidence of bilateral hydronephrosis at the age of six weeks. She had been on antibiotic prophylaxis up to now. Ultrasound scan of the brain had been normal.

On examination, she was febrile, drowsy but her anterior fontanelle was normal. Her blood pressure was $70 / 50 \mathrm{mmHg}$. The bladder was palpable and anus was patulous. She had effective limb movements against resistance.

Her blood and urine cultures were sterile. She was treated empirically with intravenous ceftriaxone for possible meningitis. Lumbar puncture done later was normal. Few days later she developed pyuria and the repeat urine culture became positive for E. coli and antibiotics were changed to intravenous Amikacin according to the sensitivity pattern. Ultrasound scan of the abdomen confirmed acute renal parenchymal disease and a diagnosis of acute pyelonephritis was made. The bladder was dilated and there was persistent bilateral hydronephrosis. A palpable bladder in this child with meningomyelocele was suggestive of neuropathic bladder which predisposed to this acute illness. She was referred to the paediatric surgeon and was commenced on clean intermittent catheterization to empty the bladder.

Her response to treatment was poor and she was clinically deteriorating. She had acidotic breathing. Her urine output was low and pyuria continued. She was transferred to Lady Ridgeway Hospital, Colombo for further management where she was treated for Gram negative septicaemia due to acute pyelonephritis and her condition improved. Further investigations did not show any evidence of chronic renal failure and she was transferred back to Teaching Hospital, Karapitiya.

We have planned for further evaluation of her renal tract with MCUG to assess reflux and DMSA for renal scarring. She is currently on nocturnal antibiotic prophylaxis to prevent further urinary tract infections and intermittent bladder catheterization.

\section{Discussion}

Neuropathic bladder may be detected antenatally with ultrasound examination or postnatally during neonatal examination. Later in childhood it may present as a voiding problem.

The associated defective or uncoordinated neuronal supply to the bladder may present as three major types of bladder dysfunction [1].

1. Synergistic dysfunction - due to an increased contraction of detrusor muscle.

2. Dysynergistic bladder - due to detrusor muscle and urethral sphincter incoordination.

3. Atonic dysfunction - due to lack of detrusor or sphincter activity. 
They are at a risk of developing recurrent UTI, VUR and hydronephrosis. Renal damage or failure can occur as a result of repeated infections with progressive renal scarring or as a result of obstruction caused by inability to empty the bladder. During follow up visits, each patient should have a physical examination with blood pressure measurement and growth monitoring, a urine analysis for proteinuria for early detection of deteriorating renal functions, a urine culture and ultrasound examination of the upper urinary tract. The best way to assess the bladder function is urodynamic studies which are not available in Sri Lanka.

Clean intermittent catheterization is the main stay of therapy and it should be done 3-4 times per day along with antibiotic prophylaxis to prevent UTI [1]. The other treatment options include pharmacological and surgical interventions. Pharmacotherapy includes anticholinergics for dysynergistic bladder. In the surgical approach lower urinary tract is manipulated by diversion, augmentation, bladder neck surgery or using artificial sphincter [1]. Ureteral reimplantation can be performed in patients with recurrent symptomatic UTIs despite adequate bladder drainage and antibiotic prophylaxis or in patients with persistent highgrade reflux with demonstrated renal scarring.

It is important to counsel regarding future social and psychological consequences of neuropathic bladder. The paediatricians can act as advocates to ensure adequate and smooth transition to adult services later [2].

\section{References}

1. RJM Coward, MA Saleem. The neuropathic bladder. Current Paediatrics 2001; 11: 135-142.

2. K Price, U Burder. Bowel and bladder management in children with disabilities. Current Paediatrics 2001; 11: $143-148$. 